\title{
Eficacia antimicrobiana del cemento Portland y el Mineral Trióxido Agregado frente a Enterococcus faecalis y Candida albicans
}

\author{
Antimicrobial efficacy of Portland cement and Mineral Trioxide Aggregate against Enterococcus \\ faecalis and Candida albicans \\ Eficácia antimicrobiana do cimento Portland e do Agregado Trióxido Mineral contra Enterococcus
}

faecalis e Candida albicans

Recibido: 26/01/2022 | Revisado: 03/02/2022 | Acepto: 05/02/2022 | Publicado: 09/02/2022

Eduardo Quea Cahuana

ORCID: https://orcid.org/0000-0001-7893-3845

Escuela Universitaria de Posgrado de la Universidad Nacional Federico Villarreal, Perú

E-mail: eduardodent@gmail.com

Wiliam Ramirez Mesías

ORCID: https://orcid.org/0000-0002-9854-1010

Facultad de Odontología de la Universidad de San Martín de Porres, Perú

E-mail: wiliamramirez15@gmail.com

María del Carmen Manrique Coras

ORCID: https://orcid.org/0000-0003-2065-0230

Facultad de Odontología de la Universidad de San Martín de Porres, Perú

E-mail: manrique.mariadelcarmen@gmail.com

Silvana Anduaga Lescano

ORCID: https://orcid.org/0000-0001-7154-2921

Facultad de Odontología de la Universidad de San Martín de Porres, Perú E-mail: Silvi_dental@hotmail.com

Javier Basilio Galvez

ORCID: https://orcid.org/0000-0002-2050-0534

Facultad de Odontología de la Universidad de San Martín de Porres, Perú

E-mail: andresbg2819@gmail.com

Shilla Anchelia Ramirez

ORCID: https://orcid.org/0000-0002-3822-0047

Facultad de Odontología de la Universidad de San Martín de Porres, Perú

E-mail: anchelia_r@hotmail.com

Brando Mellado Alfaro

ORCID: https://orcid.org/0000-0002-5226-3027

Facultad de Odontología de la Universidad de San Martín de Porres, Perú

E-mail: jeffer33@hotmail.com

\section{Resumen}

El objetivo de este estudio fue revisar la literatura disponible sobre las características del cemento Portland y el Mineral Trióxido Agregado con énfasis en su actividad antimicrobiana frente a los patógenos más prevalentes en la reinfección endodóntica. Los cementos Portland y el Mineral Trióxido Agregado, ambos a base de silicato de calcio, son utilizados en diversos ámbitos de la terapia odontológica, sobre todo en los procedimientos endodónticos, ya que estimulan los mecanismos de biomineralización. Están en contacto con las estructuras dentales, hueso y tejido conectivo, debido a ello, se enfrentan a un desafío adicional respecto a sus propiedades antibacterianas y antifúngicas contra diversos microorganismos como el Enterococcus faecalis y Candida albicans; los cuales son resistentes a los procedimientos convencionales de desinfección, sobre todo por su capacidad de formar biopelículas y estar asociados a los fracasos de tratamientos de conductos. En la revisión realizada se evidenció que en general el cemento Portland y el Mineral Trióxido Agregado tienen propiedades antimicrobianas similares y presentan actividad antimicrobiana frente a Enterococcus faecalis y Candida albicans.

Palabras clave: Mineral trióxido agregado; Cemento Portland; Enterococcus faecalis; Candida albicans.

\begin{abstract}
The objective of this study was to review the available literature on the characteristics of Portland cement and Mineral Trioxide Aggregate with emphasis on their antimicrobial activity against the most prevalent pathogens in endodontic reinfection. Portland cements and Mineral Trioxide Aggregate, both based on calcium silicate, are used in various areas of dental therapy, especially in endodontic procedures, as they stimulate biomineralization mechanisms. They
\end{abstract}


are in contact with dental structures, bone and connective tissue, therefore, they face an additional challenge regarding their antibacterial and antifungal properties against various microorganisms such as Enterococcus faecalis and Candida albicans; which are resistant to conventional disinfection procedures, especially due to their ability to form biofilms and are associated with root canal treatment failures. In the review carried out, it was shown that in general Portland cement and Mineral Trioxide Aggregate have similar antimicrobial properties and present antimicrobial activity against Enterococcus faecalis and Candida albicans.

Keywords: Mineral trioxide aggregate; Portland cement; Enterococcus faecalis; Candida albicans.

\section{Resumo}

O objetivo deste estudo foi revisar a literatura disponível sobre as características do cimento Portland e do Agregado Trióxido Mineral com ênfase em sua atividade antimicrobiana frente aos patógenos mais prevalentes na reinfecção endodôntica. Os cimentos Portland e Agregado Trióxido Mineral, ambos à base de silicato de cálcio, são utilizados em diversas áreas da terapia odontológica, principalmente em procedimentos endodônticos, pois estimulam mecanismos de biomineralização. Eles estão em contato com estruturas dentárias, osso e tecido conjuntivo, devido a isso, enfrentam um desafio adicional em relação às suas propriedades antibacterianas e antifúngicas contra diversos microrganismos como Enterococcus faecalis e Candida albicans; que são resistentes aos procedimentos convencionais de desinfecção, principalmente por sua capacidade de formar biofilmes e estarem associados a falhas no tratamento endodôntico. Na revisão realizada, foi demonstrado que em geral o cimento Portland e o Agregado Trióxido Mineral possuem propriedades antimicrobianas semelhantes e apresentam atividade antimicrobiana contra Enterococcus faecalis e Candida albicans.

Palavras-chave: Mineral trióxido agregado; Cimento Portland; Enterococcus faecalis; Candida albicans.

\section{Introducción}

El deficiente sellado a nivel apical y coronal de una pieza dental genera un ecosistema favorable a la proliferación bacteriana, las cuales son formadoras de biopelículas, además de ello, el sistema inmunológico es incapaz de actuar frente a los microorganismos patógenos presentes en el conducto radicular. Debido al sellado apical deficiente los microorganismos se nutren a través de los vasos sanguíneos presentes en esa zona, del mismo modo la pérdida del sellado a nivel coronal resulta en la invasión de más bacterias que ingresan al conducto (Ducret et al., 2017).

La infección microbiana del canal radicular conlleva a la reacción del tejido periapical. Durante la primera etapa de la infección la dentina es el tejido que ofrece resistencia a la invasión. La reacción inflamatoria generada por los microorganismos restringe la circulación sanguínea hacia la pulpa, limitando su capacidad de respuesta, provocando un daño pulpar irreversible y la progresión hacia una necrosis pulpar (Cooper et al., 2017).

El grado de inflamación y la sintomatología periapical está en relación con la cantidad de células microbianas en el conducto, grado de virulencia, así como la respuesta del huésped. La biopelícula microbiana puede ser formada tanto en los túbulos dentinarios como en las paredes del conducto, siendo su profundidad muy variable. Se han aislado más de 500 especies bacterianas en las infecciones endodónticas. En las infecciones primarias están presentes con mayor frecuencia las bacterias anaerobias Gram negativas mientras que en las infecciones secundarias la microflora predominante está integrado por cocos y bastones anaerobios facultativos Gram negativos (estreptococo, enterococo, peptoestreptococo y actinomyces). Las infecciones resistentes han sido asociadas generalmente con el Enterococos faecalis y Candida albicans (Janini et al., 2021). Si bien es cierto, los antibióticos pueden reducir de forma eficaz la inflamación en los casos de periodontitis apical aguda o crónica, no pueden eliminarla del todo, debido a que no pueden alcanzar a las bacterias presentes en el sistema de conductos (Prada et al., 2019).

Un material de relleno endodóntico ideal debería eliminar las bacterias residuales y prevenir la reinfección después del tratamiento químico-mecánico y la obturación del conducto radicular (Kapralos et al., 2018). En la búsqueda de este material ideal se ha propuesto el uso de los cementos a base de silicato de calcio como el cemento Portland y el Mineral Trióxido Agregado (MTA), los cuales cumplen con varias de las características mencionadas al ser un cemento de naturaleza hidráulica y ser muy versátil, es empleado en diversas aplicaciones clínicas con reportes favorables de su eficacia y biocompatibilidad. El polvo de estos cementos está compuesto principalmente de silicato dicálsico y tricalcico, al mezclarse 
con el agua, genera hidróxido de calcio y silicato de calcio hidratado, la mezcla forma un gel coloidal que posteriormente se solidifica (Dawood et al., 2017).

Se requiere conocer el potencial microbiano de los cementos Portland y MTA debido a que su acción frente a patógenos resistentes aún no es del todo claro y es cuestionable. Aunque presentan un $\mathrm{pH}$ altamente alcalino que le confiere la capacidad de inhibir bacterias y hongos, algunos reportes refieren que este no es suficiente (ElReash et al., 2019).

El objetivo de este estudio fue revisar la literatura disponible sobre las características del cemento Portland y el Mineral Trióxido Agregado con énfasis en su actividad antimicrobiana frente Enterococcus faecalis y Candida albicans.

\section{Metodología}

Se realizó una revisión de la literatura sobre la eficacia antimicrobiana del cemento Portland y el Mineral Trióxido Agregado frente a Enterococcus faecalis y Candida albicans. Para ello se consultaron las bases de datos: Pubmed, Scielo, Scoopus y Google Scholar utilizando diversos operadores booleanos. La estrategia de búsqueda mediante los Mesh y Decs fue la siguiente: (Mineral trioxide aggregate OR MTA cement OR MT aggregate OR aggregate ProRoot OR ProRoot aggregate OR OrthoMTA OR RetroMTA OR MTA-Fillapex) AND (Portland cement OR White Portland cement OR Gray Portland cement) AND (antimicrobial activity OR antibacterial activity OR antifungical activity OR antibiofilm activity) AND (Enterococcus faecalis OR E.faecalis OR Candida albicans OR C. albicans). Además, se realizaron búsquedas adicionales de forma manual en las revistas electrónicas: Journal of Endodontics e International Endodontic Journal. Como criterios de inclusión sólo se consideraron artículos en idioma inglés y fueron considerados los estudios específicamente relacionados con el tema de revisión de los últimos 5 años hasta la actualidad. La metodología y estructura de la extracción de datos se realizó a base a la realizada por AlShwaimi et al. (2016).

\section{Resultados y Discusión}

\section{Principales microorganismos resistentes en la infección endodóntica \\ Enterococcus faecalis}

Se ha evidenciado la presencia de esta bacteria en infecciones intrahospitalarias, ello debido al contacto de pacientes con las manos de los trabajadores de salud y los equipos médicos que se utilizan, los cuales actúan como reservorios, en este contexto se le atribuye a este microorganismo diversas infecciones asociadas, tales como endocarditis, bacteriemia, infecciones del tracto urinario, meningitis y otras formas de infecciones sistémicas y locales (Said et al., 2021).

En ese sentido, dentro de las diversas hipótesis que tratan de explicar la presencia de esta bacteria en la cavidad bucal, dos de ellas son las que han tenido mayor aceptación. La primera refiere al Enterococcus como colonizador primario, presente antes del proceso de necrosis y la segunda lo describe como un colonizador oportunista durante y posterior al tratamiento de conducto. El proceso de colonización primaria explica la presencia del Enterecoccus faecalis como agente etiológico de la caries dental, aunque esta hipótesis resulta poco probable debido a que esta bacteria tiene una frecuencia diez veces menor que las bacterias frecuentemente asociadas a la caries dental, por lo que se hace difícil atribuirle la infección pulpar a través de mecanismos de colonización primaria (Chenicheri et al., 2017). Por otro lado, la segunda hipótesis, plantea la colonización de la pulpa durante el tratamiento endodóntico o posterior a él, mediante la pérdida de sellado coronal sobre todo con la restauración temporal o permanente de larga data (Alghamdi \& Shakir, 2020).

La tasa de éxito de los tratamientos de conductos varía entre 86 a 98\% (Prada et al., 2019), sin embargo, la infección periapical persistente constituye uno de los principales motivos del fracaso endodóntico (Alghamdi \& Shakir, 2020) . Diferentes especies de microorganismos han sido detectados en las piezas dentarias, sin embargo, el Enterococcus faecalis es el 
microorganismo más frecuente en los conductos radiculares infectados y en los casos de retratamiento de periodontitis apical, alcanzando una prevalencia de 24 a 70\% (Karayasheva \& Radeva, 2017). El Enterococcus faecalis es un anaerobio facultativo Gram positivo posee mecanismos de virulencia que le permiten sobrevivir al tratamiento endodóntico convencional, llegando a invadir los túbulos dentinarios y al colágeno (Kapralos et al., 2018).

Presenta una elevada tolerancia ecológica, llegando a afrontar condiciones muy adversas debido a su polimorfismo genético (Nayyar et al., 2021). Varios autores han demostrado que esta especie bacteriana presenta también resistencia al hipoclorito de sodio a concentraciones por encima de 5\%, del mismo modo puede presentar resistencia al peróxido de hidrógeno, etanol, y radiación ultravioleta en ausencia de nutrientes durante períodos prolongados además de poseer la capacidad de multiplicarse a temperaturas entre 10 y $45^{\circ} \mathrm{C}$ (Dioguardi et al., 2018).

Diversas investigaciones han comparado la frecuencia del Enterococcus faecalis tanto en tratamiento endodónticos primarios, como en tratamientos de conductos fallidos (infección secundaria). Algunos de ellos correlacionaron la presencia del Enterococcus en mayor medida con la infección persistente que con la infección primaria, mientras otros estudios no encontraron diferencias entre ambos tipos de infecciones (Prada et al., 2019). La elevada patogenicidad del Enterococcus faecalis y las infecciones recurrentes que provoca, se debe fundamentalmente su capacidad de formar biopelículas, lo que la hace resistente a una serie de antibióticos (Garg et al., 2021), debido en una gran medida a su capacidad de formar biopelículas, los cuales se constituyen como agregados de microorganismos densos adheridos a una superficie, rodeados de una matriz polimérica formada por polisacáridos, proteínas y ADN extracelular (Swimberghe et al., 2019). La resistencia antibiótica del Enterococcus faecalis puede deberse a un componente genético de la bacteria, sin embargo, también se presenta porque los medicamentos no pueden alcanzar a las bacterias albergadas en el interior de la matriz del biofilm, actuando sólo a nivel de las capas superficiales de la película, donde luego de un tiempo, tras la disminución del efecto antibacteriano del agente administrado, las bacterias sobrevivientes proliferan formando nuevas capas de la biopelícula (Alghamdi \& Shakir, 2020). Por otro lado, el sistema inmune se encuentra incapaz de actuar frente a la bacteria, pues ella se encuentra adherida en el complejo sistema de conducto radicular. Frente a estos factores la terapia convencional no ha demostrado ser del todo eficaz, es por ello que recientes investigaciones proponen tratamientos innovadores aún no utilizados clínicamente, como el empleo de bacteriófagos, los cuales son efectivos frente a la biopelícula y a las bacterias resistentes a diversos fármacos (El-Telbany et al., 2021).

\section{Candida Albicans}

En la literatura científica se ha determinado extensamente el rol de las bacterias como causante de la periodontitis apical, sin embargo, en el proceso de colonización del conducto radicular intervienen también otros microorganismos, los cuales aún no han sido detallados ampliamente, tal es el caso de los virus, bacteriófagos, arqueas y hongos. Se ha establecido que los hongos tienen una alta prevalencia en la cavidad bucal de personas sanas y están presentes con mayor frecuencia en personas con inmunidad comprometida (Hou et al., 2022). Se le ha encontrado también en las infecciones del conducto radicular, siendo significativo su papel en la periodontitis apical. La prevalencia de especies fúngicas en el conducto infectado resulta muy variable. Algunos meta-análisis han encontrado una prevalencia del orden de 7,5\% (I. F. Persoon et al., 2017). En otro estudio (Bernal-Treviño et al., 2018) se reportó que la prevalencia de levaduras, independientemente del tipo de infección, primaria o persistente, fue del orden de $36 \%$. En la infección del conducto radicular los hongos más frecuentemente identificados fueron del género Cándida, seguidos de Saccharomyces, Aspergillus y Rhodotorula (I. F. Persoon et al., 2017). Un meta-análisis realizado por Mergoni et al. (2018) reportó que la prevalencia general de Candida en las infecciones del conducto radicular fue de $8,20 \%$, siendo la Candida albicans la especie aislada más frecuente reportada en los estudios; otras especies de Candida descritas en la misma revisión fueron Candida tropicalis, Candida kefyr, Candida parapsilosis, Candida 
glabrata, Candida krusei, Candida dubliniensis, Candida guilliermondii y Candida etchellsii. Bernal-Treviño et al. (2018) encontró una prevalencia C. albicans de 26\%. Por otro lado, Abraham et al. (2020) reportó que la prevalencia de Candida albicans fue de $5.6 \%$.

Cabe mencionar que, cierta literatura científica sugiere al componente geográfico como un factor influyente en la prevalencia de agentes infecciosos endodónticos. En ese sentido, se ha reportado la prevalencia de Candida albicans más alta en poblaciones de África $(24,82 \%)$ comparada con estudios en poblaciones de Asia, Europa, Norteamérica y Sudamérica $(10.56 \%, 4.62 \%, 4.67 \%$ y $8.57 \%$, respectivamente) (Mergoni et al., 2018).

La Candida albicans es capaz de adaptarse a condiciones adversas del ambiente endodóntico, incluso en un medio escaso de nutrientes como el conducto radicular luego de la desinfección y conformación del mismo, además es capaz de crear persistencia en un medio alcalino, en ese sentido, se ha reportado que este hongo es resistente a los medicamentos intraconducto de uso común. Aunque este microorganismo se desarrolla perfectamente en un ambiente aerobio, se ha reportado que también es capaz de adaptarse y formar biofilms en un medio anaerobio (Moazami et al., 2020).

Dentro de los mecanismos de virulencia de la Candida albicans se atribuye como factor predominante su capacidad por formar biofilms altamente estructurados, un biofilm está constituido por un conjunto de microorganismos formados en comunidad y adheridos a una superficie rodeada de una matriz extracelular, esta característica le confiere su capacidad de crear resistencia antes los agentes químicos y físicos aplicados durante el tratamiento endodóntico (Esteki et al., 2021).

Otro mecanismo de virulencia atribuida a la Candida albicans es su capacidad de sintetizar enzimas hidrolíticas, las cuales producen alteraciones a los tejidos perirradiculares, entre ellas tenemos a la aspartil-proteinasa, aminopeptidasas, colagenasas, glucosaminidasas, hialuronidasa, condroitin sulfatasa y fosfatasa alcalina, los cuales tienen la capacidad de generar degradación de las proteínas de la matriz extracelular, y generar efectos colagenolíticos en la dentina (Pereira et al., 2018).

Los hongos pueden desarrollarse independientes o en comunidad con las bacterias del conducto radicular, sin embargo, al interactuar ambos microorganismos puede resultar en el incremento de su virulencia. provocando un ambiente ecológico resistente a los tratamientos endodónticos convencionales, en esa línea Persoon et al. (2017) propusieron establecer la relación existente entre bacterias y hongos en infecciones primarias del conducto radicular, en dicha investigación confirmaron la correlación del micobioma y bacterioma infeccioso del conducto radicular en relación a la presencia de bacterias y hongos de tipo acidogénicos, sin embargo, no pudieron precisar si ello afecta el éxito del tratamiento endodóntico.

Debido a la alta prevalencia y virulencia tanto de la Candida albicans como del Enterococcus faecalis en los conductos infectados, múltiples investigaciones han estudiado diversos mecanismos de desinfección durante el proceso de tratamiento endodóntico en infecciones primarias o en retratamientos de conductos, así por ejemplo, se han planteado la aplicación de productos naturales alternativos como el chitosan o el propóleo en el tratamiento de endodoncia, específicamente frente al Candida albicans (Ganan et al., 2021), asimismo Diogo et al. (2017) propusieron el uso de la terapia fotodinámica antimicrobiana contra las biopelículas de Enterococcus faecalis y Candida albicans. Con ese mismo objetivo, Zhou et al. (2021) propusieron la eficacia de nanopartículas de plata frente a cepas de Candida albicans.

La resistencia que presentan ambos microorganismos descritos y principalmente su capacidad para formar biofilms, constituyen un reto en la terapia endodóntica en su intento de controlar y eliminar los microorganismos presentes en el sistema de conductos.

\section{Eficacia antimicrobiana del cemento Portland frente a Enterococcus faecalis y Candida albicans}

El cemento Portland es usado en la industria de la construcción, tiene una presentación de polvo fino producido por la trituración del cemento Clinker. Es considerado de naturaleza hidráulica, está compuesto de $65 \%$ de cal, 20\% de sílice, $10 \%$ de 
alúmina y óxido férrico y $5 \%$ de otros componentes. La cal está compuesta de óxido de calcio y magnesio (Primathena et al., 2021).

El proceso de producción consiste en la molienda de minerales, arcilla y cal, las cuales son calcinadas a $1400{ }^{\circ} \mathrm{C}$ produciendo cambios físicos y químicos en las materias primas. El “clíncker” resultante de este proceso se pulveriza y se añade 2 a 3\% de yeso para retardar el fraguado (Osiro et al., 2018). Entre los componentes resultantes encontramos al silicato dicálcico [Ca2SiO4], silicato tricálcico [Ca3SiO5], aluminato tricálcico [Ca3A12O6], sulfato de calcio [CaSO4, yeso], y aluminoferrita de tetracalcio [4CaOAl 2O3Fe2O3] (Dawood et al., 2017).

El Minera Trióxido Agregado (MTA) es un cemento a base de disilicato de calcio, empleado en diversos procedimientos odontológicos, sobre todo como reparador de tejido en perforaciones. En el mercado actual, su costo es muy elevado, a pesar que se conoce su alta eficacia en una serie de tratamientos aún no es accesible a todos los niveles de atención de salud, es por ello que diversos autores han investigado materiales alternativos como posibilidad de reemplazo o sustitución. En esa búsqueda, diversos autores han estudiado y comparado las propiedades químicas del MTA y el cemento Portland, reportando en sus conclusiones que ambos cementos están constituidos por los mismos elementos, a excepción del óxido de bismuto que le es añadido al MTA para proporcionarle propiedades de radiopacidad (Primathena et al., 2021). En ese sentido, el primer estudio que consideró como posibilidad de comparación al cemento Portland y el MTA fue el realizado por Estrela et al. (2000), quien comparó los componentes químicos de los materiales a través de un análisis de fluorescencia de rayos X, concluyendo que el cemento Portland contiene los mismos elementos químicos del MTA a excepción del óxido de bismuto de este último, similar conclusión obtuvo Osiro et al. (2018) quien a través de un análisis de disfracción de rayos x comparó al cemento Portland y MTA determinando que no hubo diferencias significativas entre ambos materiales.

A partir de la confirmación de que los componentes químicos del MTA son muy similares al del cemento Portland las investigaciones se centraron en establecer sus propiedades físicas y biológicas en el afán de considerar a este último como material de reemplazo.

Una investigación concluyó que el cemento Portland tiene las mismas propiedades de biocompatibilidad que el MTA (Qutieshat et al., 2019). Otros estudios al realizar una comparación y análisis de las propiedades físico químicas del cemento Portland y el MTA concluyeron que ambos presentan propiedades similares (Osiro et al., 2018).

Con el fin de mejorar diversas propiedades del cemento Portland y en su afán de proponerlo como sustituto del MTA o incluso superar los beneficios de este último, diversos investigadores le han añadido compuestos adicionales, entre los que figuran agentes radiopacificadores, aceleradores, sustancias antimicrobianas sintéticas o naturales, entre otros, sin que estos influyan en sus propiedades fisicoquímicas y biológicas (Ganan et al, 2021; Li y Coleman, 2019). En una investigación, de Souza et al. (2017) reportó que la adición del 15\% de carbonato de bismuto como radiopacificador del cemento Portland no produjo cambios significativos en sus propiedades fisicoquímicas y biológicas en comparación con el MTA, excepto por el tiempo de fraguado, y puede considerarse un posible sustituto del MTA.

Por otro lado, los cementos a base de silicato de calcio como el MTA y el cemento Portland presentan una actividad antimicrobiana con resultados contradictorios, ya que varias investigaciones mencionan que tiene un efecto limitado (Sahia, 2018; Shin, 2017). Para mejorar sus propiedades antimicrobianas se ha propuesto la adición de una serie de agentes, los cuales no alteraron significativamente sus propiedades fisicoquímicas ni biológicas. Tal como los demuestran los estudios realizados al añadirle componentes antimicrobianos como monómeros de amonio cuaternario (Yang et al., 2018), Aloe Vera (Henrique Borges et al., 2017), nanopartículas de óxido de zirconio y óxido de niobium, óxido de zirconio, nanopartículas de plata entre otros (Song \& Ge, 2019).

La actividad antimicrobiana del cemento Portland se debe fundamentalmente a que cuando es expuesto al agua, forma hidróxido de calcio, el cual induce al incremento del pH a través de la disociación de iones de calcio e hidroxilo, aunque esta es 
la principal explicación para establecer el mecanismo de acción antimicrobiana, existen organismos endodónticos que pueden eliminarse en condiciones ajenas a las variaciones del pH (Janini et al., 2021). Diversas investigaciones han evaluado al cemento Portland y la mayoría de ellas han demostrado que este material presenta actividad antibacteriana y antifúngica principalmente frente a Enterococcus faecalis y Candida albicans respectivamente, además que su eficacia para eliminar microorganismos es muy parecida al MTA, sin embargo, algunos otros estudios no han demostrado estas propiedades o consideran que sus efectos antimicrobianos son limitados. Así, por ejemplo, Miyagak et al. (2006) al evaluar la eficacia antimicrobiana del cemento Portland y el MTA a través del método de difusión en agar, no evidencia efecto antimicrobiano frente a Enterococcus faecalis ni Candida albicans en ninguno de los dos materiales. A similar conclusión llegó otra investigación (Melo et al., 2015), la cual reportó que ni el MTA ni el cemento Portland tuvieron efecto antibacteriano frente al Enterococcus faecalis, pero si inhibieron, mediante la prueba de difusión en agar, el crecimiento de otro tipo de bacterias cariogénicas como el Lactobacillus acidophilus.

Otro estudio (Asgary et al., 2009) indicó que no hubo diferencias entre el cemento Portland y MTA respecto a las propiedades antibacterianas frente a Enterococcus faecalis en ninguno de los tres tiempos investigados, concluyendo que ambos presentan actividad antibacteriana, aunque no mayor que el hidróxido de calcio. Otra investigación (Miyagak et al., 2006), al evaluar al cemento Portland puro y compararlo con la adición a este de diversos radiopacificadores, encontró que sus propiedades antibacterianas y antifúngicas no se vieron afectadas y que todas las especies microbianas fueron inhibidas por los cementos evaluados. Una investigación (Tanomaru-Filho et al., 2007) también reportó que al evaluar la actividad antimicrobiana del MTA blanco y gris y el cemento Portland blanco y gris frente a Enterococcus faecalis y Candida albicans no se evidenciaron diferencias entre ambos materiales, presentando un efecto limitado contra dichas cepas en comparación con otros cementos evaluados en dicho estudio. Como hasta ahora se ha visto, las propiedades antimicrobianas del MTA y cemento Portland si bien son similares en cuanto a sus características físicas, químicas y biológicas, son limitadas en su efecto antimicrobiano si son comparados con otros materiales, es por ello que algunos investigadores en el intento de mejorar esta propiedad han propuesto añadir ciertos compuestos a estos cementos a fin de mejorar su eficacia frente a bacterias y hongos. Así, por ejemplo, en un estudio (Guerreiro-Tanomaru et al., 2014) se añadieron micro y nanopartículas de óxido de zirconio al cemento Portland y al MTA a fin de mejorar sus propiedades frente a Enterococcus faecalis, Candida albicans y otros microorganismos, los resultados indicaron que al añadir estas partículas al MTA se evidenció una mejora en las propiedades antimicrobianas, no ocurriendo lo mismo con el cemento Portland. Otras investigaciones (Nam, 2017) han añadido al cemento Portland nanopartículas de plata para analizar su eficacia antimicrobiana encontrando que esta promueve la inhibición frente a las bacterias estudiadas, además de promover sus propiedades biomecánicas. En un estudio (Nashaat et al., 2019) se comparó la eficacia antimicrobiana frente a Enterococcus faecalis del MTA y cemento Portland, tanto en su estado original, como en sus estados nanoparticulados encontrando que el cemento Portland tuvo mejor eficacia que el MTA en ambas presentaciones.

\section{Eficacia antimicrobiana del Mineral Trióxido Agregado frente a Enterococcus faecalis y Candida albicans}

Torabinejad desarrolló en la década de los 90's un cemento a base de silicato conocido como Mineral Trióxido Agregado (MTA), el cual fue aprobado por la Administración de Alimentos y Medicamentos de los Estados Unidos (FDA) en 1998 y comercializado bajo la marca ProRoot® MTA (Dentsply Tulsa Dental, Johnson City, TN, EE. UU.) (Parirokh et al., 2018) Debido a sus altas propiedades y versatilidad en la aplicación de diversos procedimientos, el MTA se ha convertido en el sustituto de hidróxido de calcio $\mathrm{Ca}(\mathrm{OH}) 2$, como lo demuestran estudios clínicos, esta necesidad de reemplazo de hidróxido de calcio se debe a mejorar la calidad del tejido duro inducido por el material y a que este puede producir debilitamiento de la dentina conllevando a un riesgo de fractura a nivel de la raíz (Al-Hiyasat et al., 2021).

El polvo del MTA está compuesto de cemento Portland y óxido de bismuto como agente radiopacificador, además, 
presenta pequeñas cantidades de óxido de silicio ( $\mathrm{SiO} 2)$, óxido de calcio ( $\mathrm{Ca} 2)$, óxido de magnesio $(\mathrm{MgO})$, sulfato de potasio (K2SO4) y sulfato de sodio (Na2SO4), estas finas partículas hidrofílicas de polvo se mezclan con el agua destilada que está incluido en el kit de MTA (Parirokh et al., 2018).

Diferentes estudios han examinado los efectos antimicrobianos del MTA con resultados discordantes (Pelepenko et al., 2021). Múltiples investigaciones reportan que los cementos a base de silicato de calcio como el MTA presentan un efecto antibacteriano y antifúngico discreto o limitado, así por ejemplo en una investigación Farrugia et al. (2017) examinó la actividad antimicrobiana del MTA ProRoot, frente al Enterococcus faecalis, sometiendo este material al contacto con sangre, agua y heparina, utilizando como indicador de la actividad antimicrobiana el test de difusión agar, el test de contacto directo y el test de infección intratubular, encontrando que el MTA en contacto con sangre tuvo una actividad antimicrobiana limitada al ser medido con el test de contacto directo y el test de infección intratubular, asimismo, concluye que el MTA tuvo una reducción significativa de la actividad antimicrobiana después de los siete días. En el mismo sentido, Sahia et al. (2018) realizó un estudio in vitro para determinar la eficacia antimicrobiana de diferentes selladores endodónticos (sellador a base de resina, sellador a base de óxido de zinc eugenol, sellador a base de hidróxido de calcio y mineral trióxido agregado - MTA) frente al Enterococcus faecalis, a través del método de difusión en agar, encontrando que el MTA fue el cemento de menor eficacia antibacteriana, decayendo aún más su efectividad conforme transcurría el tiempo.

El MTA no presentó eficacia antibacteriana contra Enterococcus faecalis, Estafilococo aureus, Bacillus subtilis, Escherichia coli u otras bacterias anaerobias en un estudio realizado por Torabinejad et al. (1995). En otra investigación Shin et al. (2017), al comparar el efecto antibacteriano entre el MTA ProRoot y un nuevo MTA de fraguado rápido frente a cepas de Enterococcus faecalis y Estreptococo mutans a través de la técnica de difusión en agar, no se evidenció efecto antibacteriano en ninguno de los dos materiales evaluados después de 24 y 48 horas. Asimismo, Morgental et al. (2011) al evaluar diferentes selladores endodónticos a base de silicato de calcio, entre los que figuraban el MTA blanco (Angelus, Londrina, PR, Brazil) y Endo CPM Sealer reportó que estos últimos no presentaron efecto antibacteriano frente al Enterococcus faecalis.

Los estudios de actividad antimicrobiana presentados hasta ahora en nuestra revisión han evidenciado un efecto escaso de los cementos a base de silicato de calcio como el MTA, sin embargo, se conoce que la actividad antibacteriana de este material se debe fundamentalmente a sus altos valores de $\mathrm{pH}$ y a la liberación de hidróxido de calcio, el cual produce la desnaturalización de proteínas del medio (Queiroz et al., 2021). La alcalinización del medio ocurre a través de la disociación de los iones de calcio y los iones hidróxido cuando el material entra en contacto con el agua, sin embargo; el Enterococcus faecalis puede sobrevivir a un $\mathrm{pH}$ cercano a 12.5 (Rojas et al., 2021).

Tal como se mencionó, los reportes existentes son contradictorios, ello puede deberse a la naturaleza de la fuente bacteriana, la diferencia entre cepas, la cantidad de bacterias inoculadas, el tiempo de incubación, la actividad metabólica de los microorganismos evaluados, además de las propias características del material investigado, tales como el tamaño molecular, solubilidad y difusión de los materiales a través del medio agar acuoso además de la propia metodología de determinación de la eficacia antibacteriana, entre otros (Silva et al., 2014) .

En una reciente revisión sistemática AlShwaimi et al. (2016) refieren, en contraparte a lo que hasta ahora se ha manifestado en nuestra revisión, que todas las investigaciones incluidas en su estudio presentaron actividad antimicrobiana positiva sobre cultivos de Enterococcus faecalis, aunque califica de moderados su nivel de evidencia. Otra investigación (Koruyucu et al., 2015) reporta que al comparar el MTA Angelus, Biodentin y Dycal frente a cepas de Enterococcus faecalis a través del test de contacto directo, el MTA evidenció la mejor actividad antibacteriana, siendo el Biodentine muy similar a él. En dicha investigación también se menciona que, para determinar las características antimicrobianas de un material, tradicionalmente se emplean el test de difusión en agar y el test de contacto directo, presentando este último mayores ventajas debido a un mejor control de los factores de confusión, además de tener la capacidad de evaluar materiales frescos, 
inmediatamente después de su manipulación, además de tener la capacidad de evaluar la actividad antimicrobiana de componentes insolubles. El uso de un método u otro puede variar los resultados de la investigación, hecho que se evidencia en el estudio realizado por Jafari et al. (2017) donde al evaluar el efecto antifúngico del MTA Fillapex y el cemento resinoso AH26 encontró que al aplicar el método directo el MTA Fillapex fue significativamente menor que el AH-26, sin embargo, al utilizar el método indirecto la actividad antifúngica de ambos materiales fue similar.

Debido al uso versátil y a las propiedades favorables del MTA, en los últimos años se han introducido nuevos materiales en el tratamiento endodóntico cuyo componente principal está basado en el silicato de calcio. El primer sellador endodóntico de esta nueva clase fue introducido en el año 2007 bajo el nombre de iRoot SP (Innovative Bioceramix, Vancouver, Canada) el cual presenta una composición muy similar al MTA (Donnermeyer et al., 2019). En una investigación (Primathena et al., 2021) determinaron el efecto antimicrobiano del MTA y un cemento Portland modificado, al que añadieron oxido de bismuto como radiopacificador; ambos cementos fueron enfrentados al Enterococcus faecalis a través del método de difusión en agar encontrando que los dos cementos tuvieron efecto inhibitorio.

Otro material endodóntico a base de silicato de calcio que busca mejorar algunas desventajas del MTA se presentó en el mercado bajo el nombre de EndoBinder (Binderware, Sao Carlos, SP, Brasil), según algunos reportes (Silva et al., 2014) presenta buena respuesta celular lo cual significa un mayor desarrollo de células en estado avanzado de diferenciación osteoblástica que la obtenida con el MTA, además de contar con menos reacción tisular; al ser comparado con el MTA blanco respecto a sus propiedades antimicrobianas no mostró diferencias significativas, resultando igual de eficaz frente a Enterococcus faecalis, sin embargo, frente a la Candida albicans el EndoBiner tuvo un menor efecto que el MTA a las 48 horas.

Si bien se ha reconocido al Enterococcus faecalis como la bacteria responsable de los fracasos endodónticos, como se ha mencionado, la infección del conducto se manifiesta también por la presencia de una serie de microorganismos entre los que figuran la Candida albicans, sobre todo en las infecciones persistentes debido a sus mecanismos de patogenicidad y virulencia (Yoo et al., 2020).

Se ha descrito que las propiedades antifúngicas del MTA, se deben principalmente al elevado $\mathrm{pH}$ que presenta, el cual tiene un valor inicialmente de 10.2 que se eleva a 12.5 luego de tres horas después de mezclado. Es por ello necesario considerar el tiempo de fraguado del MTA, ya que este se presenta en un periodo relativamente largo de 3 horas, durante este tiempo, todavía se producen reacciones químicas del material mezclado, por lo que es necesario considerar ese intervalo en el cual podría no ser efectivo (Torabinejad et al., 1995).

Las limitaciones de la presente revisión están relacionadas con la heterogeneidad de los artículos analizados, ya que, para determinar la eficacia antimicrobiana de los cementos, los investigadores utilizan distintas técnicas, agares, tiempos de evaluación y concentraciones (Tabla 1 y 2), por lo que la capacidad de comparación de los estudios podría verse limitada y debe realizarse considerando esos aspectos. 
Research, Society and Development, v. 11, n. 3, e2311326172, 2022

(CC BY 4.0) | ISSN 2525-3409 | DOI: http://dx.doi.org/10.33448/rsd-v11i3.26172

Tabla 1. Estudios que incluyen al MTA y cemento Portland en la evaluación de la eficacia antimicrobiana frente a Enterococcus faecalis.

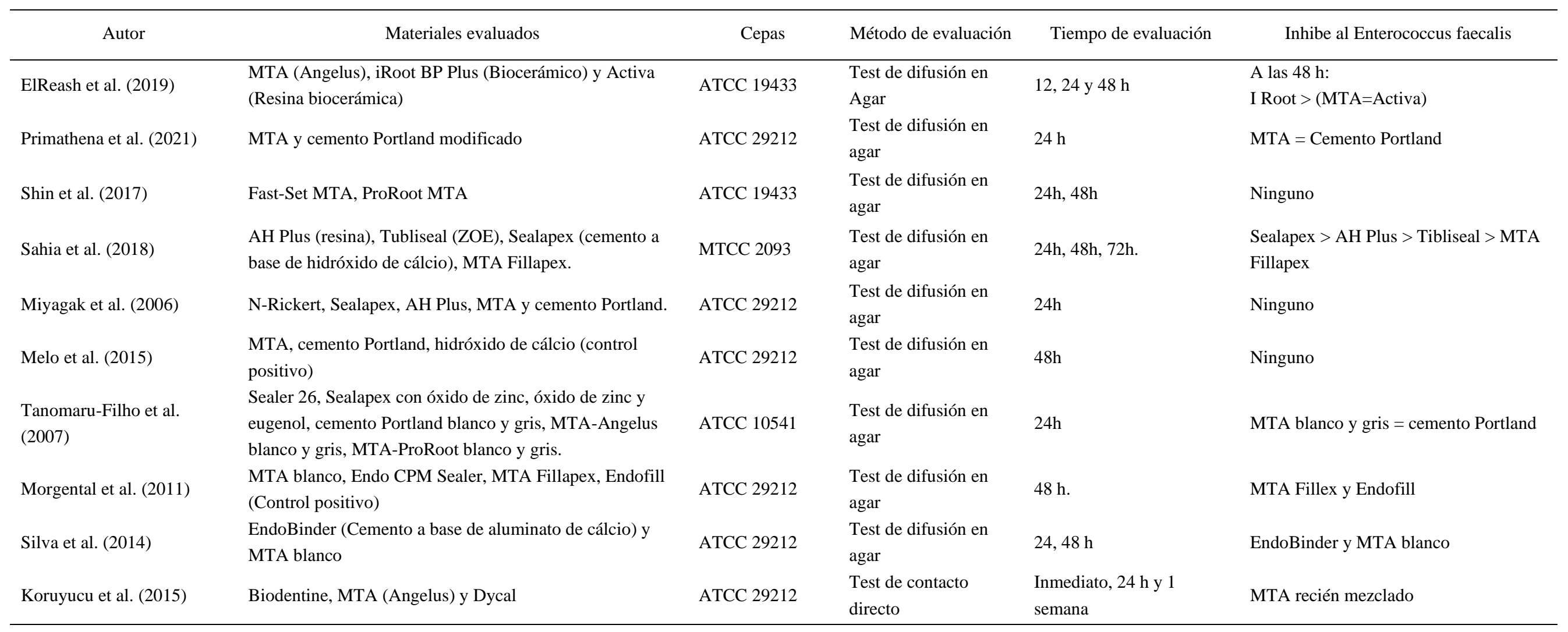

Fuente: Elaboración propia. 
Tabla 2. Estudios que incluyen al MTA y cemento Portland en la evaluación de la eficacia antimicrobiana frente a Candida albicans.

\begin{tabular}{|c|c|c|c|c|c|}
\hline Autor & Materiales evaluados & Cepas & $\begin{array}{l}\text { Método de } \\
\text { evaluación }\end{array}$ & Tiempo de evaluación & Inhibe a la Candida albicans \\
\hline ElReash et al. (2019) & $\begin{array}{l}\text { MTA (Angelus), iRoot BP Plus (Biocerámico) y Activa } \\
\text { (Resina biocerámica) }\end{array}$ & ATCC 10231 & $\begin{array}{l}\text { Test de difusión en } \\
\text { Agar }\end{array}$ & 12,24 y $48 \mathrm{~h}$ & $\begin{array}{l}\text { A las } 48 \mathrm{~h}: \\
\text { I Root }>\text { MTA > Activa }\end{array}$ \\
\hline Miyagak et al. (2006) & $\begin{array}{l}\text { N-Rickert, Sealapex, AH Plus, MTA y cemento } \\
\text { Portland. }\end{array}$ & ICB/USP 562 & $\begin{array}{l}\text { Test de difusión en } \\
\text { agar }\end{array}$ & $24 \mathrm{~h}$ & AH Plus y N-Rickert \\
\hline $\begin{array}{l}\text { Tanomaru-Filho et al. } \\
\text { (2007) }\end{array}$ & $\begin{array}{l}\text { Sealer 26, Sealapex con óxido de zinc, óxido de zinc y } \\
\text { eugenol, cemento Portland blanco y gris, MTA-Ángelus } \\
\text { blanco y gris, MTA-ProRoot blanco y gris. }\end{array}$ & ATCC 10541 & $\begin{array}{l}\text { Test de difusión en } \\
\text { agar }\end{array}$ & $24 \mathrm{~h}$ & MTA blanco y gris $=$ cemento Portland \\
\hline Silva et al. (2014) & $\begin{array}{l}\text { EndoBinder (Cemento a base de aluminato de calcio) y } \\
\text { MTA blanco }\end{array}$ & ATCC 10556 & $\begin{array}{l}\text { Test de difusión en } \\
\text { agar }\end{array}$ & $24.48 \mathrm{~h}$ & $\begin{array}{l}\text { MTA blanco > que EndoBinder a las } \\
48 \mathrm{~h}\end{array}$ \\
\hline Jafari et al. (2017) & AH 26 y MTA Fillapex & ATCC 10231 & $\begin{array}{l}\text { Test de contacto } \\
\text { directo }\end{array}$ & 24h, 48h, 72 h y 7 días & AH-26 (24h) $>$ MTA $(24 \mathrm{~h})$ \\
\hline Nurdin et al. (2021) & MTA y cemento Portland modificado & ATCC 10231 & $\begin{array}{l}\text { Test de contacto } \\
\text { directo }\end{array}$ & $24 \mathrm{~h}$ & MTA $=$ Cemento Portland modificado \\
\hline
\end{tabular}

Fuente: Elaboración propia. 
Research, Society and Development, v. 11, n. 3, e2311326172, 2022

(CC BY 4.0) | ISSN 2525-3409 | DOI: http://dx.doi.org/10.33448/rsd-v11i3.26172

\section{Conclusión}

En la revisión realizada se alcanzó el objetivo de evaluar la eficacia antimicrobiana del cemento Portland y el Mineral Trióxido Agregado. Evidenciándose que, en general el cemento Portland y el Mineral Trióxido Agregado tienen propiedades antimicrobianas similares y presentan actividad antimicrobiana frente a Enterococcus faecalis y Candida albicans.

Se sugiere realizar revisiones adicionales que evalúen las propiedades antimicrobianas de los compuestos añadidos al cemento Portland y el Mineral Trióxido Agregado.

\section{Referencias}

Abraham, S., Al Marzooq, F., Himratul-Aznita, W., Ahmed, H., \& Samaranayake, L. (2020). Prevalence, virulence and antifungal activity of C. albicans isolated from infected root canals. BMC Oral Health, 20(1).

Al-Hiyasat, A. S., El-Farraj, H. S., \& Alebrahim, M. A. (2021). The effect of calcium hydroxide on dentine composition and root fracture resistance of human teeth: An in vitro study. European Journal of Oral Sciences, 129(4), e12798.

Alghamdi, F., \& Shakir, M. (2020). The Influence of Enterococcus faecalis as a Dental Root Canal Pathogen on Endodontic Treatment: A Systematic Review. Cureus, 12(3).

AlShwaimi, E., Bogari, D., Ajaj, R., Al-Shahrani, S., Almas, K., \& Majeed, A. (2016). In Vitro Antimicrobial Effectiveness of Root Canal Sealers against Enterococcus faecalis: A Systematic Review. Journal of Endodontics, 42(11), 1588-1597.

Asgary, S., Eghbal, M. J., Parirokh, M., Ghoddusi, J., Kheirieh, S., \& Brink, F. (2009). Comparison of Mineral Trioxide Aggregate's Composition with Portland Cements and a New Endodontic Cement. Journal of Endodontics, 35(2), 243-250.

Bernal-Treviño, A., González-Amaro, A., Méndez González, V., \& Pozos-Guillen, A. (2018). Frequency of Candida in root canals of teeth with primary and persistent endodontic infections. Revista Iberoamericana de Micología, 35(2), 78-82.

Chenicheri, S., R, U., Ramachandran, R., Thomas, V., \& Wood, A. (2017). Insight into Oral Biofilm: Primary, Secondary and Residual Caries and PhytoChallenged Solutions. The Open Dentistry Journal, 11(1), 312.

Cooper, P., Chicca, I., Holder, M., \& Milward, M. (2017). Inflammation and Regeneration in the Dentin-pulp Complex: Net Gain or Net Loss? Journal of Endodontics, 43(9S), S87-S94.

Dawood, A. E., Parashos, P., Rhk, W., Reynolds, E. C., \& Manton, D. J. (2017). Calcium silicate-based cements: composition, properties, and clinical applications. Journal of investigative and clinical dentistry, 8(2)

De Souza, L., Yadlapati, M., Lopes, H., Silva, R., Letra, A., \& Elias, C. (2017). Physico-chemical and Biological Properties of a New Portland Cement-based Root Repair Material. European Endodontic Journal, 3(1).

Diogo, P., Fernandes, C., Caramelo, F., Mota, M., Miranda, I. M., Faustino, M. A. F., Neves, M. G. P. M. S., Uliana, M. P., de Oliveira, K. T., Santos, J. M., \& Gonçalves, T. (2017). Antimicrobial photodynamic therapy against endodontic Enterococcus faecalis and Candida albicans mono and mixed biofilms in the presence of photosensitizers: A comparative study with classical endodontic irrigants. Frontiers in Microbiology, 8, 498.

Dioguardi, M., Di Gioia, G., Illuzzi, G., Laneve, E., Cocco, A., \& Troiano, G. (2018). Endodontic irrigants: Different methods to improve efficacy and related problems. European Journal of Dentistry, 12(3), 459-466.

Donnermeyer, D., Bürklein, S., Dammaschke, T., \& Schäfer, E. (2019). Endodontic sealers based on calcium silicates: a systematic review. Odontology, 107(4), 421-436.

Ducret, M., Fabre, H., Celle, A., Mallein-Gerin, F., Perrier-Groult, E., Alliot-Licht, B., \& Farges, J.C. (2017). Current challenges in human tooth revitalization. Bio-Medical Materials and Engineering, 28(s1), S159-S168.

El-Telbany, M., El-Didamony, G., Askora, A., Ariny, E., Abdallah, D., Connerton, I., \& El-Shibiny, A. (2021). Bacteriophages to Control Multi-Drug Resistant Enterococcus faecalis Infection of Dental Root Canals. Microorganisms, 9(3), 1-19.

ElReash, A. A., Hamama, H., Eldars, W., Lingwei, G., Zaen El-Din, A. M., \& Xiaoli, X. (2019). Antimicrobial activity and pH measurement of calcium silicate cements versus new bioactive resin composite restorative material. BMC Oral Health 19(1), 1-10.

Esteki, P., Jahromi, M., \& Tahmourespour, A. (2021). In vitro antimicrobial activity of mineral trioxide aggregate, Biodentine, and calcium-enriched mixture cement against Enterococcus faecalis, Streptococcus mutans, and Candida albicans using the agar diffusion technique. Dental Research Journal, $18(1), 3$.

Estrela, C., Bammann, L. L., Estrela, C. R., Silva, R. S., \& Pécora, J. D. (2000). Antimicrobial and chemical study of MTA, Portland cement, calcium hydroxide paste, Sealapex and Dycal. Brazilian Dental Journal, 11(1), 3-9.

Farrugia, C., Baca, P., Camilleri, J., \& Arias Moliz, M. T. (2017). Antimicrobial activity of ProRoot MTA in contact with blood. Scientific Reports, 7(1),1-10.

Ganan, M., Lorentzen, S., Gaustad, P., \& Sørlie, M. (2021). Synergistic Antifungal Activity of Chito-Oligosaccharides and Commercial Antifungals on 
Research, Society and Development, v. 11, n. 3, e2311326172, 2022

(CC BY 4.0) | ISSN 2525-3409 | DOI: http://dx.doi.org/10.33448/rsd-v11i3.26172

Biofilms of Clinical Candida Isolates. Journal of Fungi (Basel, Switzerland), 7(9).

Garg, A., Mala, K., \& Kamath, P. (2021). Biofilm models in endodontics-A narrative review. Journal of Conservative Dentistry, $24(1), 2-9$.

Guerreiro-Tanomaru, J. M., Trindade-Junior, A., Cesar Costa, B., Da Silva, G. F., Drullis Cifali, L., Basso Bernardi, M. I., \& Tanomaru-Filho, M. (2014). Effect of Zirconium Oxide and Zinc Oxide Nanoparticles on Physicochemical Properties and Antibiofilm Activity of a Calcium Silicate-Based Material. Scientific World Journal, 2014.

Henrique Borges, A., Aguirre Guedes, O., \& Evaristo Ricci Volpato, L. (2017). Physicochemical Properties of MTA and Portland Cement after Addition of Aloe Vera. Iranian Endodontic Journal, 12(13), 312-317.

Hou, Y., Wang, L., Zhang, L., Tan, X., Huang, D., \& Song, D. (2022). Potential relationship between clinical symptoms and the root canal microbiomes of root filled teeth based on the next-generation sequencing. International Endodontic Journal, 55(1), 18-29.

Jafari, F., Jafari, S., Samadi Kafil, H., Momeni, T., \& Jamloo, H. (2017). Antifungal activity of two root canal sealers against different strains of Candida. Iranian Endodontic Journal, 12(1), 98-102.

Janini, A. C. P., Bombarda, G. F., Pelepenko, L. E., \& Marcano, M. A. (2021). Antimicrobial activity of calcium silicate-based dental materials: A literature review. Antibiotics, $10(7), 7$.

Kapralos, V., Koutroulis, A., Ørstavik, D., Sunde, P. T., \& Rukke, H. V. (2018). Antibacterial Activity of Endodontic Sealers against Planktonic Bacteria and Bacteria in Biofilms. Journal of Endodontics, 44(1), 149-154.

Karayasheva, D., \& Radeva, E. (2017). Importance of Enterococci (Enterococcus faecalis) for Dental Medicine - Microbiological Characterization, Prevalence and Resistance. International Journal of Science and Research, 6(7), 1970.

Koruyucu, M., Topcuoglu, N., Tuna, E. B., Ozel, S., Gencay, K., Kulekci, G., \& Seymen, F. (2015). An assessment of antibacterial activity of three pulp capping materials on Enterococcus faecalis by a direct contact test: An in vitro study. European Journal of Dentistry, 9(2), 240-245.

Li, Q., \& Coleman, N. (2019). Impact of Bi 2 O 3 and ZrO 2 Radiopacifiers on the Early Hydration and C-S-H Gel Structure of White Portland Cement. Journal of Functional Biomaterials, 10(4).

Melo, P. M. R. de, Sobral, A. P. V., Sampaio, G. C., Pinto, I. M. de A., \& Shinohara, N. K. S. (2015). Evaluation of cariogenic antibacterial activity of mineral trioxide aggregate and Portland cement. $R G O$ - Revista Gaúcha de Odontologia, 63(2), 181-186.

Mergoni, G., Percudani, D., Lodi, G., Bertani, P., \& Manfredi, M. (2018). Prevalence of Candida Species in Endodontic Infections: Systematic Review and Meta-analysis. Journal of Endodontics, 44(11), 1616-1625.e9.

Miyagak, D. C., de Carvalho, E. M. O. F., Robazza, C. R. C., Chavasco, J. K., \& Levorato, G. L. (2006). In vitro evaluation of the antimicrobial activity of endodontic sealers. Brazilian Oral Research, 20(4), 303-306.

Moazami, F., Gholami, A., Mehrabi, V., \& Ghahramani, Y. (2020). Evaluation of the antibacterial and antifungal effects of ProRoot MTA and nano-fast cement: An in vitro study. Journal of Contemporary Dental Practice, 21(7), 760-764.

Morgental, R. D., Vier-Pelisser, F. V., Oliveira, S. D., Antunes, F. C., Cogo, D. M., \& Kopper, P. M. P. (2011). Antibacterial activity of two MTA-based root canal sealers. International Endodontic Journal, 44(12), 1128-1133.

Nam, K. Y. (2017). Characterization and antimicrobial efficacy of Portland cement impregnated with silver nanoparticles. Journal of Advanced Prosthodontics, 9(3), 217-223.

Nashaat, Y., Ahmed, L., \& Nada, O. (2019). Comparative study of the antibacterial effect of MTA. Nano-MTA, Portland cement, and nano-Portland cement. Egyptian Dental Journal, 33(65), 701-706.

Nayyar, P., Sethi, A., Thakur, D., Khullar, S., Gayati, S., \& Adarsh, K. (2021). Antibacterial Effect of Silver Nanoparticle Gel as an Intracanal Medicament in Combination with Other Medicaments against Enterococcus faecalis: An In vitro Study. Journal of Pharmacy \& Bioallied Sciences, $13(1)$, S408.

Nurdin, D., Sari, M. I., Adang, R. A. F., Primathena, I., \& Cahyanto, A. (2021). Antifungal Effectiveness between Tricalcium Silicate-White Portland Cements Added Bi2O3 and Mineral Trioxide Aggregate Against Candida albicans. The Open Dentistry Journal, 14(1), 757-762.

Osiro, A., Kariuki, D., \& GAthece, W. (2018). Composition and particle size of mineral trioxide aggregate, portland cement and synthetic geopolymers. East African Medical Journal, 95(5), 1522.

Parirokh, M., Torabinejad, M., \& Dummer, P. M. H. (2018). Mineral trioxide aggregate and other bioactive endodontic cements: an updated overview - part I: vital pulp therapy. International Endodontic Journal, 51(2), 177-205.

Pelepenko, L. E., Saavedra, F., Tbm, A., \& others. (2021). Physicochemical, antimicrobial, and biological properties of White-MTAFlow. Clinical Oral Investigation, 25(2), 663-672.

Pereira, D., Seneviratne, C., Koga-Ito, C., \& Samaranayake, L. (2018). Is the oral fungal pathogen Candida albicans a cariogen? Oral Diseases, 24(4), 518526.

Persoon, I. F., Crielaard, W., \& Özok, A. R. (2017). Prevalence and nature of fungi in root canal infections: a systematic review and meta-analysis. International Endodontic Journal, 50(11), 1055-1066. 
Research, Society and Development, v. 11, n. 3, e2311326172, 2022

(CC BY 4.0) | ISSN 2525-3409 | DOI: http://dx.doi.org/10.33448/rsd-v11i3.26172

Persoon, Ilona F., Buijs, M. J., Özok, A. R., Crielaard, W., Krom, B. P., Zaura, E., \& Brandt, B. W. (2017). The mycobiome of root canal infections is correlated to the bacteriome. Clinical Oral Investigations, 21(5), 1871.

Prada, I., Micó-Muñoz, P., Giner-Lluesma, T., Micó-Martínez, P., Collado-Castellano, N., \& Manzano-Saiz, A. (2019). Influence of microbiology on endodontic failure. Literature review. Medicina Oral, Patología Oral y Cirugía Bucal, 24(3), e364.

Primathena, I., Nurdin, D., Hermawan, H., \& Cahyanto, A. (2021). Synthesis, Characterization, and Antibacterial Evaluation of a Cost-Effective Endodontic Sealer Based on Tricalcium Silicate-White Portland Cement. Materials, 14(2), 1-9.

Queiroz, M. B., Torres, F. F. E., Rodrigues, E. M., Viola, K. S., Bosso-Martelo, R., Chavez-Andrade, G. M., Guerreiro-Tanomaru, J. M., \& Tanomaru-Filho, M. (2021). Physicochemical, biological, and antibacterial evaluation of tricalcium silicate-based reparative cements with different radiopacifiers. Dental Materials, 37(2), 311-320.

Qutieshat, A. S., Al-Hiyasat, A. S., \& Darmani, H. (2019). Biocompatibility evaluation of Jordanian Portland cement for potential future dental application. Journal of Conservative Dentistry, 22(3), 249-254.

Rojas, B., Soto, N., Villalba, M., Bello-Toledo, H., Meléndrez-Castro, M., \& Sánchez-Sanhueza, G. (2021). Antibacterial Activity of Copper Nanoparticles (CuNPs) against a Resistant Calcium Hydroxide Multispecies Endodontic Biofilm. Nanomaterials, 11(9).

Sahia, D., Gaikwad, A., Samuel, R., Aher, G., Gulve, M., \& Kolhe, S. (2018). Antimicrobial Efficacy of Different Endodontic Sealers against Enterococcus faecalis: An In vitro Study. Journal of International Society of Preventive \& Community Dentistry, 8(2), 104.

Said, M. S., Tirthani, E., \& Lesho, E. (2021). Enterococcus Infections. Treasure Island StatPearl.

Shin, M., Chen, J. W., Tsai, C. Y., Aprecio, R., Zhang, W., Yochim, J. M., Teng, N., \& Torabinejad, M. (2017). Cytotoxicity and antimicrobial effects of a new fast-set MTA. BioMed Research International, 1-6

Silva, E. J. N. L., Herrera, D. R., Rosa, T. P., Duque, T. M., Jacinto, R. C., Gomes, B. P. F. D. A., \& Zaia, A. A. (2014). Evaluation of cytotoxicity, antimicrobial activity and physicochemical properties of a calcium aluminate-based endodontic material. Journal of Applied Oral Science, $22(1), 61-67$.

Song, W., \& Ge, S. (2019). Application of Antimicrobial Nanoparticles in Dentistry. Molecules 24(6), 1033.

Swimberghe, R., Coenye, T., De Moor, R., \& Meire, R. (2019). Biofilm model systems for root canal disinfection: a literature review. International Endodontic Journal, 52(5), 604-628.

Tanomaru-Filho, M., Tanomaru, J. M. G., Barros, D. B., Watanabe, E., \& Ito, I. Y. (2007). In vitro antimicrobial activity of endodontic sealers, MTA-based cements and Portland cement. Journal of Oral Science, 49(1), 41-45.

Torabinejad, M., Hong, C. U., Ford, T. R. P., \& Kettering, J. D. (1995). Antibacterial effects of some root end filling materials. Journal of Endodontics, 21(8), 403-406.

Yang, Y. W., Yu, F., Zhang, H. C., Dong, Y., Qiu, Y. N., Jiao, Y., Xing, X. D., Tian, M., Huang, L., \& Chen, J. H. (2018). Physicochemical properties and cytotoxicity of an experimental resin-based pulp capping material containing the quaternary ammonium salt and Portland cement. International Endodontic Journal, 51(1), 26-40.

Yoo, Y., Kim, A., Perinpanayagam, H., Han, S., \& Kum, K. (2020). Candida albicans Virulence Factors and Pathogenicity for Endodontic Infections. Microorganisms, 8(9), 1-18.

Zhou, L., Zhao, X., Li, M., Lu, Y., \& Ai, C. (2021). Antifungal activity of silver nanoparticles synthesized by iturin against Candida albicans in vitro and in vivo. Applied Microbiology and Biotechnology, 105(9), 3759-3770. 